\title{
Analysis of Criteria for Determining a TNT Equivalent
}

\author{
Robert Panowicz* - Marcin Konarzewski - Michał Trypolin \\ Military University of Technology, Department of Mechanical Engineering, Poland
}

The ConWep method is one of the most frequently used methods of modelling the effects of a blast wave impulse on a structure. The main goal of the study was to assess a TNT equivalent used in this approach. Six different methods to calculate this parameter for different explosive materials and charge shapes were analysed in this paper. The results error was lower than $20 \%$ only when a TNT equivalent based on comparing the pressure impulses was used. This error can be lower only for spherical charges. In addition, for the tested distances $(25 \mathrm{~cm}$ to $40 \mathrm{~cm}$ ), TNT equivalents with an error margin of $5 \%$ were assessed.

Keywords: TNT equivalent, ConWep, blast wave

Highlights

- For assessing the effects of the blast wave on the structure, a ballistic pendulum was used.

- $\quad$ TNT equivalent was determined with the use of six different methods for two explosive materials: Semtex A1 and Composition $B$.

- $\quad$ Numerical analyses were conducted with ConWep and ALE approaches.

- Validation of the numerical model was performed.

- $\quad$ For the tested distances, TNT equivalents with an error margin of $5 \%$ were assessed.

\section{INTRODUCTION}

Numerical analyses used to predict phenomena are always challenging. In the case of modelling the construction loads caused by blast waves, the challenges are related to the shape of the blast impulse and the method of describing its effects on the structure.

In the simplest cases, the dynamic load of the blast wave is realized through the use of a triangular pressure wave affecting the structure [1]. In such a situation, the maximal value of the wave corresponds to the maximal value of the pressure obtained from experimental tests, and the area under the experimental curve corresponds to the value of a pressure impulse. This method allows expressing the influence of the positive part of the blast wave.

For determining the parameters of the blast wave, experimental dependencies described by Henrych [2] and Sadovskiy [3] are often used.

Kingery and Bulmash [4], based on experimental data conducted with explosive charges made of trinitrotoluene (TNT), proposed dependencies describing the change of the pressure for reduced mass and distance. The results of their studies were implemented in many computer programs [5] to [7], also known as conventional weapon effects (ConWep). Apart from assessing the parameters of the blast wave, they also enable the determination of the influence of the pressure impulse on the structure. The ConWep method is one of the most frequently used methods of modelling the effects of a blast wave impulse on the structure.

Difficulty appears when an explosive material other than TNT or with a shape other than spherical is used. When using other explosive materials, a socalled TNT equivalent is used. The TNT equivalent parameter is a value representing how much the mass of the charge needs to be multiplied by to obtain the same blast wave propagation. There is no definite method for calculating the TNT equivalent. Cooper used TNT equivalence based on a comparison of the velocities of detonation in accordance with the following equation [8]:

$$
T N T e q=D_{e x p}^{2} / D_{T N T}^{2},
$$

where $D$ is the velocity of detonation, subscripts exp and TNT are the studied explosive and TNT explosive, respectively.

One of the most frequently used methods of calculating the TNT equivalent is to determine it from the heat of detonation [9]:

$$
T N T e q=Q_{\text {exp }} / Q_{T N T},
$$

where $Q$ is the heat of detonation.

This method is recommended by the unified facilities criteria manuals (UFC) [10] in experiments where free air explosions are studied.

Eq. (2) was modified by Locking [9]:

$$
N T e q=\frac{Q_{\text {exp }}}{(1-d) Q_{T N T}+m \cdot Q_{e x p}},
$$


where $d=0.76862$ represents the Y-intercept of the straight curve of the heat of detonation TNT equivalent graph, and $m=0.7341$ the angular coefficient of the straight curve of the heat of detonation TNT equivalent graph.

Another equation for calculating the TNT equivalence was proposed in study [11] and is presented below:

$$
T N T e q=\frac{p_{C J}+20.9}{40}
$$

where $p_{C J}$ is the pressure of detonation [GPa].

The equations presented so far for determining the TNT equivalence were based on the available or easily obtainable material parameters. The more advanced methods shown below require more calculations or experimental tests to be conducted.

Another method is based on choosing, by trial and error, the TNT mass in such a way that the pressure values at a specified distance are equal [12] and [13]. Then, TNT equivalent is expressed by the following equation:

$$
\text { TNTeq }=\left(\frac{Z_{\text {exp }}}{Z_{T N T}}\right)^{3},
$$

where $Z=R / \sqrt[3]{W}$ is the reduced distance from the explosive, $R$ the distance from the explosive, and $W$ the mass of the explosive charge.

The authors of study [12], when calculating the TNT equivalent, used an equation related to the equivalent pressure impulse at the specified distance:

$$
\text { TNTeq }=\left(\frac{W_{\text {exp }}}{W_{T N T}}\right)_{I},
$$

where $W_{T N T}$ is the mass of TNT causing the equivalent, positive pressure impulse at the same distance as the mass $W_{\text {exp }}$ of the material in question, and $I$ the impulse.

When using non-spherical charges, it is often assumed that in comparably greater distances the pressure distribution would be similar to the spherical explosive where the detonation occurred centrally. Owing to this fact, the shape of the explosive is often omitted. The shape is indirectly referenced in Eqs. (5) and (6).

It results from the analysis of the detonation process and pressure wave propagation that the TNT equivalence value depends on: detonation speed, pressure, detonation energy and the number of moles of gaseous detonation products [14]. However, many experimental studies show that the adoption of a constant value of this parameter allows for the accurate determination of pressure impulse parameters, although they may depend on the determination method [15]. In the range of reduced distances $\mathrm{Z}$ from $8 \mathrm{~m} / \mathrm{kg}^{1 / 3}$ to $27 \mathrm{~m} / \mathrm{kg}^{1 / 3}$, the TNT equivalent TATP is equal to 0.7 and 0.55 for determination based on Eqs. (5) and (6), respectively [16]. The results presented in [17] showed that in the case of the C-4 explosive, the TNT equivalent is a nonlinear function of the reduced distance and a logarithm of the equivalent. In contrast, Rigby and Sielicki demonstrated, based on experimental research and numerical analysis, that the TNT equivalent value depends on the mass of the explosive charge mass [18]. However, the constant value of TNT equivalent for PE4 can be assumed to be suitable for both pressure and impulse equivalence, and that this value is also invariant of distance from the explosive.

Another aspect associated with TNT equivalent is a large spread of the published values of this parameter [19]. The typical spread of these values causes a $20 \%$ to $30 \%$ difference in blast wave parameter values.

The aim of the study is verification of the methods presented earlier Eqs. (1) to (6) for assessing the TNT equivalent. On the basis of the acquired TNT equivalent values, the mass of the TNT charges used in the ConWep numerical analyses was determined. In the next step, the numerical results of the ballistic pendulum tilt, which is the effect of blast wave interaction, determined with the use of 2 methods, were compared. The first method, validated and used as a reference, uses an arbitrary Lagrangian-Eulerian (ALE) with fluid-structure interaction (FSI) approach [18] and [6]. The other one, which is approximate, uses a ConWep approach with the determined earlier TNT mass.

The finite element method with an explicit integration was used to perform the numerical analyses. For this purpose, the authors used the LSDyna software [6].

The numerical analyses were conducted for spherical and cylindrical explosives with a mass of 50 $\mathrm{g}$, with the explosives placed from $25 \mathrm{~cm}$ to $45 \mathrm{~cm}$ from the pendulum.

Before the proper analyses, the validation process of the ballistic pendulum numerical model, created with the use of the ALE with an FSI approach, was conducted. A good agreement with the experimental tests was achieved.

In Chapter 1 of the paper, the results of experimental tests used for validating the numerical model shown in Chapter 2 are presented. The results of the numerical analyses are presented in Chapter 3, and then briefly characterized in the summary. 


\section{EXPERIMENTAL TESTS}

For assessing the effects of the blast wave on the structure, a ballistic pendulum was used. The test rig consists of the following parts (Fig. 1):

- double T-bar 220,

- two $300 \mathrm{~mm} \times 300 \mathrm{~mm}$ steel plates, $10 \mathrm{~mm}$ thick, welded to the ends of the T-bar,

- eight distancing elements with a length of $200 \mathrm{~mm}$ constructed of threaded rods with a diameter of $24 \mathrm{~mm}$,

- two $300 \mathrm{~mm} \times 300 \mathrm{~mm}$ steel, removable plates, $20 \mathrm{~mm}$ thick,

- four steel ropes with a diameter of $5 \mathrm{~mm}$,

- a frame consisting of closed steel profiles.

The dimensions of each part were selected in a way that allows the rig to work in the elastic range.

During the experiment, the frontal part of the pendulum was loaded dynamically by the blast wave caused by the detonation of the explosive. For the test, Semtex A1 cylindrical explosives with a mass of $50 \mathrm{~g}$ and with $\mathrm{L} / \mathrm{D}=1$ ratio were used. The charges were placed on a light Styrofoam stand at a distance of $30 \mathrm{~cm}$ and $35 \mathrm{~cm}$ from the pendulum. The distance was measured from the front plate of the pendulum to the frontal part of the explosive.

For registering the tilt of the pendulum, fast camera Phantom V12 with tagged points (Fig. 2) was used. Recording of the process was conducted with no less than $2000 \mathrm{fps}$. The tilt of the pendulum, as registered by the camera, is shown in Fig 4. For the case in which the distance between the pendulum and the charge was $30 \mathrm{~cm}$, the maximum tilt was equal to $75 \mathrm{~mm}$. For the $35 \mathrm{~cm}$ distance, the maximum tilt was $64 \mathrm{~mm}$.

\section{NUMERICAL MODEL}

In the numerical model, only the core parts of the pendulum were modelled: ropes, double T-bar, a)

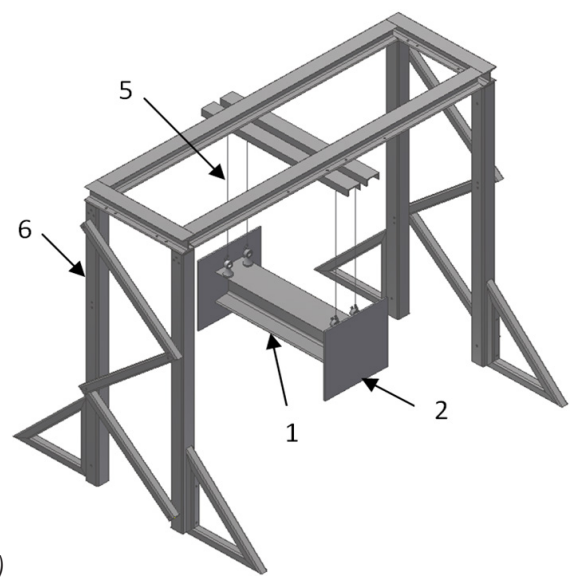

b)

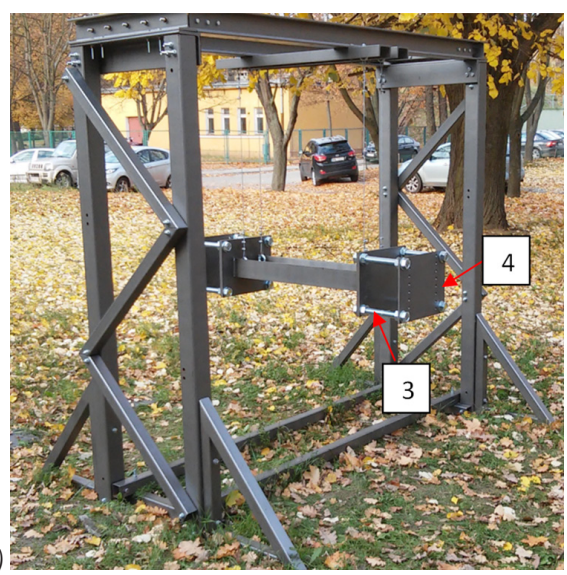

Fig. 1. a) Diagram of the test setup; and b) test rig; 1 double T-bar, 2 steel plate, 3 distancing element, 4 steel, removable plate, 5 rope, 6 frame
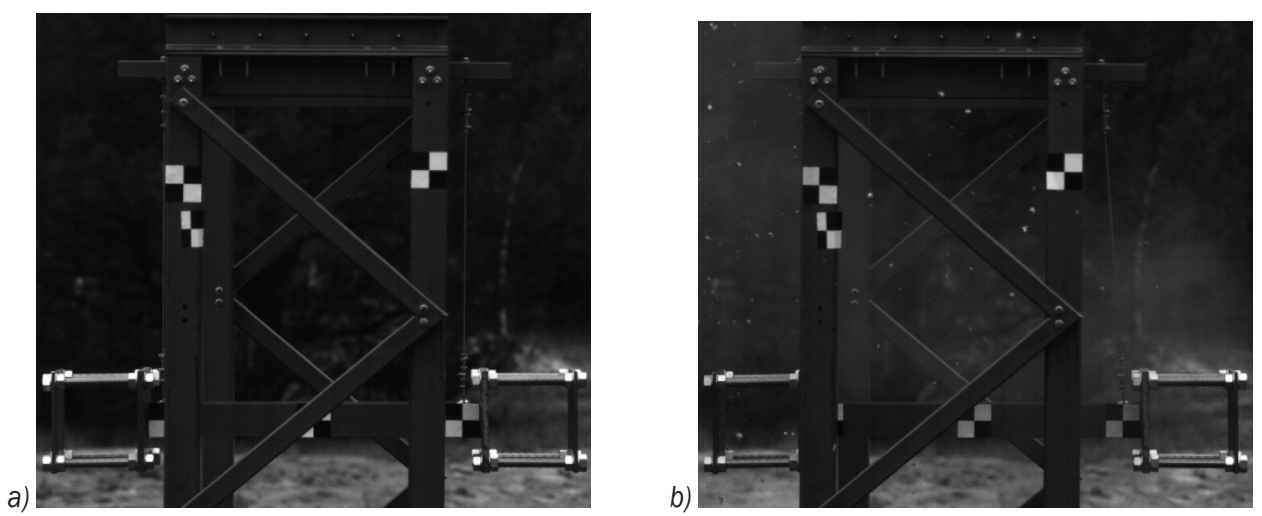

Fig. 2. Test rig during the experimental tests; a) initial position, b) tilt during the test with $50 \mathrm{~g}$ Semtex $A 1$ cylindrical explosive $L / D=1$, placed in the distance $d=30 \mathrm{~cm}$ from the pendulum 
plates and distancing elements (Fig. 3). The model consists of 4464 solid finite elements with 1 point of integration and 408 1D bar elements (ropes). For mechanical properties, an elastic material model was used, as shown in Table 1.

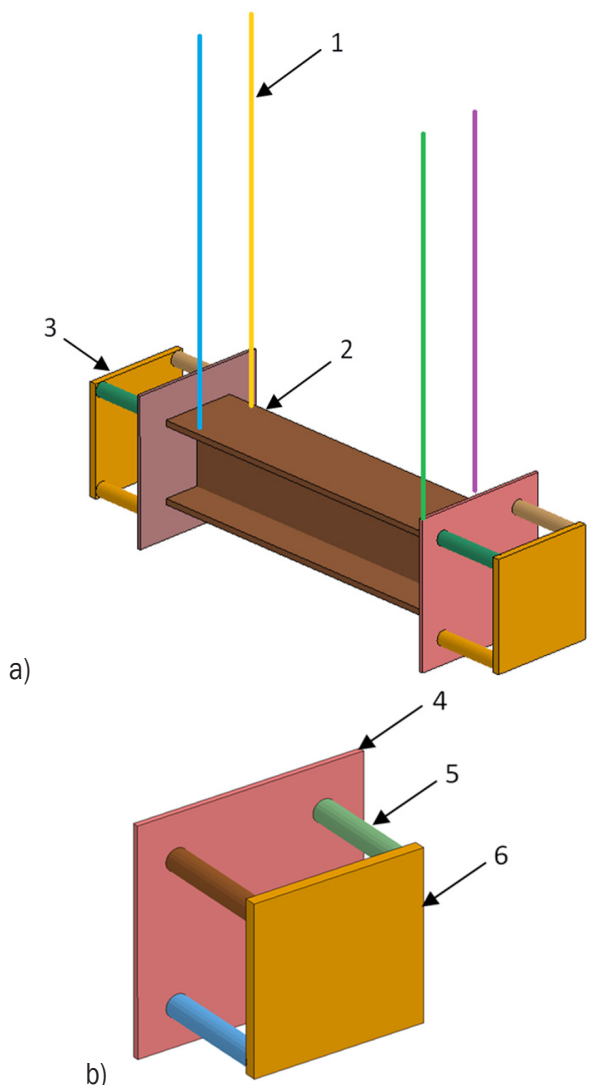

Fig. 3. a) Numerical model of the ballistic pendulum; b) close-up of the front part of the pendulum; 1 steel ropes, 2 double T-bar,

3 counter-weight, 4 front plate, 5 distancing elements, 6 removable front plate

The influence of the pressure wave caused by detonation of the explosive charge was realized using two methods. The first, which is considered as a referential one, uses the ALE method and the FSI coupling. This method is often used in many studies because it is characterized by high accuracy [20] and [21].

Table 1. Material parameters for elastic constitutive relationship

\begin{tabular}{llcc}
\hline Parameter & Description & Unit & Value \\
\hline$\rho$ & Density & $\mathrm{kg} / \mathrm{m}^{3}$ & $7.89 \mathrm{E} 3$ \\
\hline$E$ & Young modulus & $\mathrm{Pa}$ & $2.1 \mathrm{E} 11$ \\
\hline$v$ & Poisson's ratio & - & 0.3 \\
\hline
\end{tabular}

The air domain was simulated using the MieGruneisen equation:

$$
p=p_{0}+\gamma \rho E_{\text {int }},
$$

where $p$ is pressure, $p_{0}$ initial pressure, $\gamma$ Gruneisen coefficient, $E_{\text {int }}$ internal energy.

Jones-Wilkins-Lee (JWL) equation was used for describing the behaviour of detonation products:

$$
p=A\left(1-\frac{\omega}{R_{1} V}\right)^{-R_{1} V}+B\left(1-\frac{\omega}{R_{2} V}\right)^{-R_{2} V},
$$

where $V=\rho_{0} / \rho, \rho_{0}$ is initial density, $\rho$ density of detonation products, and $A, B, R_{1}, R_{2}, \omega$ are constants.

Material parameters used in the JWL equation are taken from literature [22] and are presented in Table 2.

The validation process of the developed numerical model was based on a comparative analysis of the profiles of experimental and numerical tilts of the ballistic pendulum. When comparing the results of the experimental test and the numerical analysis using the ALE with the FSI approach (Fig. 4), a high compatibility is visible. The maximal error in the considered cases was not higher than $2 \%$ between the tilt obtained from the numerical analysis and the tilt obtained from the experimental test for the variant with the distance of $30 \mathrm{~cm}$ between the pendulum and the charge and $3 \%$ with the distance of $35 \mathrm{~cm}$. In both cases depicted, the tilt curves representing the experimental tests lie above the curves representing the numerical tests results.

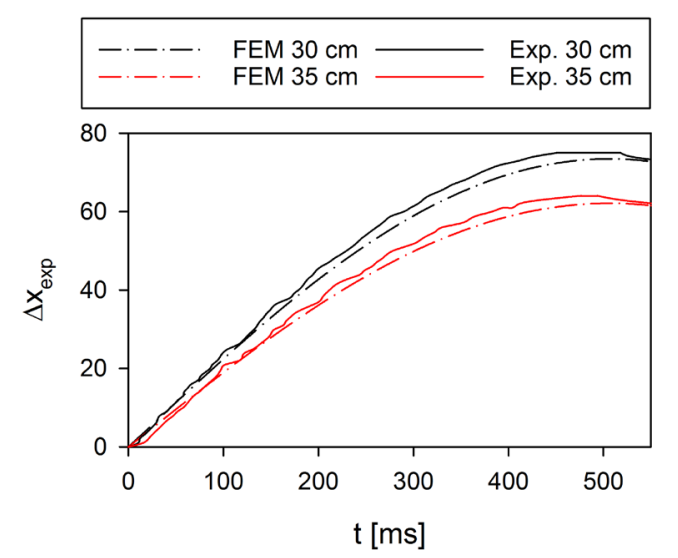

Fig. 4. Tilt of the ballistic pendulum for $50 \mathrm{~g}$ Semtex A1 cylindrical explosive (solid line - experiment, dashed line - FEM numerical results) for the distances $30 \mathrm{~cm}$ and $35 \mathrm{~cm}$ from the pendulum

\section{RESULTS AND DISCUSSION}

The numerical tests were conducted using two frequently used explosive materials, Comp B and Semtex A1. For those materials, the influence of 
both the shape of the charge and the distance from the pendulum on the maximal tilt of the pendulum was also analysed. The tested charges, spherical and cylindrical, had shapes close to spherical $(\mathrm{L} / \mathrm{D}=1)$.

As mentioned earlier, the results of analyses performed using the ALE with the FSI approach are considered as a reference.

The mass of the TNT used in the ConWep method calculations was derived from the TNT equivalent Eqs. (1) to (6) for $50 \mathrm{~g}$ explosive charges made from Comp B and Semtex A1 and located at a distance of $25 \mathrm{~cm}$ to $45 \mathrm{~cm}$ from the pendulum ballistic. This corresponds to a distance reduced from $0.68 \mathrm{~m} / \mathrm{kg}^{1 / 3}$ to $1.22 \mathrm{~m} / \mathrm{kg}^{1 / 3}$. The maximum pressure values and pressure impulses needed to determine the TNT equivalents on the basis of Eqs. (5) and (6), respectively, were obtained from the numerical comparisons of the parameters (pressure, impulse) obtained from the numerical analysis using the ALE approach [15].

In the first step, analyses were performed for spherical and cylindrical explosives, made from Comp $\mathrm{B}$ and Semtex A1, recording pressure and impulse values at distances from $25 \mathrm{~cm}$ to $45 \mathrm{~cm}$, every $5 \mathrm{~cm}$. A number of similar numerical analyses were carried out for TNT charges with different weights. For the initial values, the average TNT mass, obtained using Eqs. (1) to (3), was assumed. The analyses were completed at the time when the relative difference between the pressure or impulse value obtained for the TNT load and test material was less than $2 \%$.

The results presented in Figs. to 8 show maximal pendulum tilts obtained by ALE with FSI calculations and ConWep with TNT masses presented in Table 3 and distances from $25 \mathrm{~cm}$ to $45 \mathrm{~cm}$ (distance reduced from $0.68 \mathrm{~m} / \mathrm{kg}^{1 / 3}$ to $\left.1.22 \mathrm{~m} / \mathrm{kg}^{1 / 3}\right)$.

When analysing the curves showing the maximal tilt of the pendulum in function of the distance of the charge from the pendulum as calculated using numerical simulation with the use of ALE method with FSI coupling, a clear influence of both the shape and the distance can be observed (Figs. 5 to 8 ALE). The influence is the lowest for the cylindrical charge made of Semtex A1.

The tilt of the pendulum for charges made of Comp B in the cases that used the ConWep method and Eq. (4) overestimates the tilt values regardless of a charge shape (Figs. 5 and 6). In all other cases, the curves are close to each other.

For cylindrical charges at the shortest considered distance $(25 \mathrm{~cm})$, the most significant difference between two values is $40 \mathrm{~mm}$ (33\%). In the case with the longest distance between the charge and the pendulum, the values decrease to $18 \mathrm{~mm}$ (29\%). Similar results were acquired for spherical charges. After excluding method 4, the values drop significantly. For cylindrical charges, the values decrease to $14 \%$ and $6 \%$ for $25 \mathrm{~cm}$ and $45 \mathrm{~cm}$, respectively.

Table 2. Explosive materials parameters [16]

\begin{tabular}{llccc}
\hline Parameter & Description & Unit & Semtex & Comp B \\
\hline$\rho$ & Density & {$\left[\mathrm{kg} / \mathrm{m}^{3}\right]$} & 1400 & 1710 \\
\hline$D$ & Detonation speed & {$[\mathrm{m} / \mathrm{s}]$} & 7200 & 7980 \\
\hline$p_{C J}$ & C-J pressure & {$[\mathrm{GPa}]$} & 28 & 29.5 \\
\hline$A$ & Explosive material constant & {$[\mathrm{GPa}]$} & 609 & 524.2 \\
\hline$B$ & Explosive material constant & {$[\mathrm{GPa}]$} & 12.95 & 7.678 \\
\hline$R_{1}$ & Explosive material constant & - & 4.5 & 4.2 \\
\hline$R_{2}$ & Explosive material constant & - & 1.4 & 1.1 \\
\hline$\omega$ & Explosive material constant & - & 0.25 & 0.34 \\
\hline
\end{tabular}

For both types of charges, the curve representing the ALE with the FSI method has a similar shape, however, the degree of the slope is different. This causes the cases with shortest distance between the pendulum and the charge to have a lower pendulum tilt than the ConWep method, and the longer distance cases to have a higher pendulum tilt than the ConWep method, regardless of the equation used, with the exception of the overall overestimation of Eq. 4. The

Table 3. Summary of numerical tests

\begin{tabular}{cccccccccc}
\hline \multirow{2}{*}{ Symbol } & Equation & \multicolumn{7}{c}{ TNT mass corresponding to the $50 \mathrm{~g}$ explosive charge and the TNT equivalent value } \\
\cline { 3 - 10 } & $\begin{array}{c}\text { Spherical } \\
\text { Semtex A1 [g] }\end{array}$ & TNT equiv. & $\begin{array}{c}\text { Cylindrical } \\
\text { Semtex A1 [g] }\end{array}$ & TNT equiv. & $\begin{array}{c}\text { Spherical } \\
\text { Comp B [g] }\end{array}$ & TNT equiv. & $\begin{array}{c}\text { Cylin-drical } \\
\text { Comp B [g] }\end{array}$ & TNT equiv. \\
\hline A & 1 & 54 & 1.08 & 54 & 1.08 & 66.3 & 1.326 & 66.3 & 1.326 \\
\hline B & 2 & 20.25 & 0.405 & 20.25 & 0.405 & 55.6 & 1.112 & 55.6 & 1.112 \\
\hline C & 3 & 91.5 & 1.83 & 91.5 & 1.83 & 63 & 1.26 & 63 & 1.26 \\
\hline D & 4 & 48 & 0.96 & 48 & 0.96 & 90.4 & 1.808 & 90.4 & 1.808 \\
\hline E & 5 & 80.1 & 1.602 & 82.3 & 1.646 & 64.15 & 1.283 & 70.8 & 1.416 \\
\hline F & 6 & 65.5 & 1.31 & 65.15 & 1.303 & 59.6 & 1.192 & 59.55 & 1.191 \\
\hline
\end{tabular}




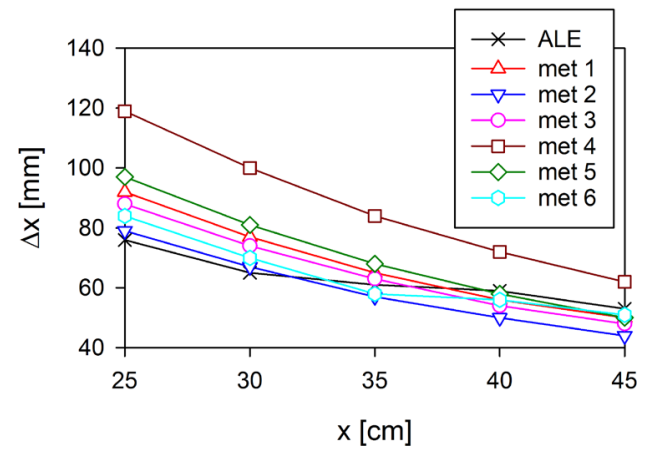

Fig. 5. Maximal tilt of the ballistic pendulum when affected by a blast wave caused by detonation of $50 \mathrm{~g}$ Comp B cylindrical explosive charge for different methods

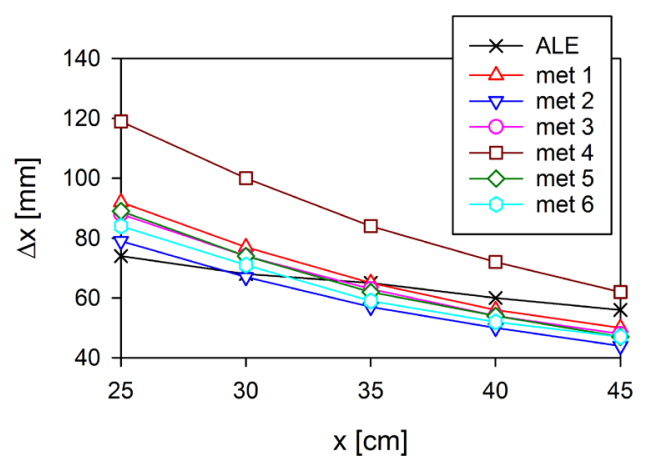

Fig. 6. Maximal tilt of the ballistic pendulum when affected by a blast wave caused by detonation of $50 \mathrm{~g}$ Comp B spherical explosive charge for different methods

lowest mean error $(10.5 \%)$ for the pendulum tilt was achieved with Eq. (3) for cylindrical charges. Both Eqs. (3) and (6) achieve slightly above $11 \%$ of error for spherical charges.

The curves representing Semtex A1 material are more apart from each other (Figs. 7 and 8), with values of $50 \mathrm{~mm}$ for a $25 \mathrm{~cm}$ distance and $36 \mathrm{~mm}$ for a $45 \mathrm{~cm}$ distance for both cylindrical and spherical charges.

The method based on the heat of detonation in Eq. (2) for this material results in underestimated tilt values.

The character of the curves presented in Figs. 7 and 8 is similar; therefore, the mean errors of the tilt equal to $6.3 \%$ and $10 \%$ for cylindrical and spherical charges, respectively, are lower when compared to Comp B material results. The highest accuracy in both cases is achieved with the equation based on comparing pressure impulses in Eq. (6).

In all considered cases, the dispersion of pendulum tilt values for shorter distances are

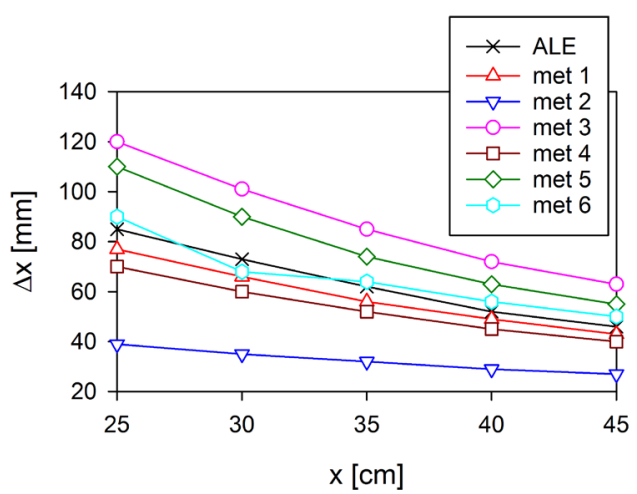

Fig. 7. Maximal tilt of the ballistic pendulum when affected by a blast wave caused by detonation of $50 \mathrm{~g}$ Semtex A1 cylindrical explosive charge for different methods

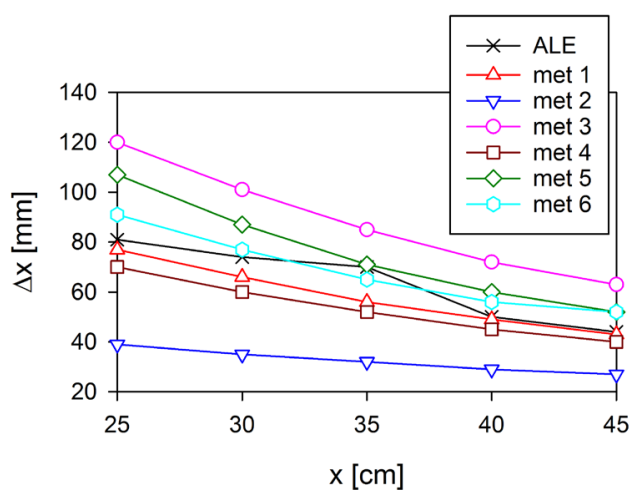

Fig. 8. Maximal tilt of the ballistic pendulum when affected by a blast wave caused by detonation of $50 \mathrm{~g}$ Semtex $\mathrm{A} 1$ spherical explosive charge for different methods

significantly higher than for longer distances (Figs. 5 to 8 ).

Table 4 shows the values of obtained TNT equivalents with maximal tilt errors not higher than $2 \%$ for Semtex A1 and Comp B for both spherical and cylindrical charges. These values were obtained on the basis of an optimization process in which the objective function was to minimize the relative pendulum tilt, and the variable was TNT mass in the ConWep approximation in Eq. (9). As a reference, the pendulum tilt value obtained using the ALE with the FSI method was used in the calculations. Matlab software was used in the analyses.

$$
\delta=\min \frac{\Delta x-\Delta x_{A L E}}{\Delta x_{A L E}},
$$

where $\Delta x$ is maximal pendulum tilt obtained for ConWep approximation for a given mass of TNT, $\Delta x_{A L E}$, maximal pendulum tilt obtained for ALE with the FSI approach for the given explosives. 
Table 4. TNT equivalence values for spherical and cylindrical charges made of Comp B and Semtex A1 based on optimization tests

\begin{tabular}{|c|c|c|c|c|c|}
\hline \multirow{2}{*}{$\begin{array}{l}\text { Distance } \\
\text { [cm] }\end{array}$} & \multirow{2}{*}{$\begin{array}{l}\text { Reduced distance } \\
{\left[\mathrm{m} / \mathrm{kg}^{1 / 3}\right]}\end{array}$} & \multicolumn{2}{|c|}{ Comp B } & \multicolumn{2}{|c|}{ Semtex A1 } \\
\hline & & Cylinder & Sphere & Cylinder & Sphere \\
\hline 25 & 0.68 & 1.05 & 1.05 & 1.22 & 1.11 \\
\hline 30 & 0.81 & 1.05 & 1.112 & 1.25 & 1.3 \\
\hline 35 & 0.95 & 1.21 & 1.27 & 1.26 & 1.474 \\
\hline 40 & 1.09 & 1.35 & 1.4 & 1.15 & 1.08 \\
\hline 45 & 1.22 & 1.4 & 1.55 & 1.2 & 1.08 \\
\hline
\end{tabular}

\section{CONCLUSIONS}

The conducted tests clearly show that only a pressure impulse method for assessing the TNT equivalent yields errors lesser than $20 \%$, which is consistent with the results of work [15]. Lower error values can be achieved only with spherical charges. In other cases, it should be taken into account that the error will float around values no lower than $40 \%$.

Based on the compared data, TNT equivalents with $5 \%$ error were assessed for spherical and cylindrical charges made of Comp B and Semtex A1. The value is a sum that results from comparing the ALE with the FSI methods to the experimental data and between the error of the ConWep method and the ALE with the FSI method.

\section{ACKNOWLEDGMENTS}

This article was co-funded by The National Centre for Research and Development - project number DOBRBIO4/022/13149/2013.

\section{REFERENCES}

[1] Syed, Z.I., Mohamed, O.A., Rahman, S.A. (2016). Non-linear finite element analysis of offshore stainless steel blast wall under high impulsive pressure loads. Procedia Engineering, vol. 145, p. 1275-1282, D0l:10.1016/j.proeng.2016.04.164.

[2] Henrych J. (1979). The Dynamic of Explosion and Its Use. Elsevier Science Ltd., Prague.

[3] Sadovskiy M. A., (2004). Mechanical effects of air shockwaves from explosions according to experiments. Geophysics and Physics of Explosion. Nauka Press, Moscow. (in Russian)

[4] Kingery, C.N., Bulmash, G. (1984). Airblast Parameters from TNT Spherical Air Burst and Hemispherical Surface Burst. BRT Technical Report ARBRL-TR-02555. U.S. Army Armament Research and Development Center, Aberdeen.

[5] Hyde, D.W. (1991). Conventional Weapons Program (ConWep). U.S. Army Waterways Experimental Station, Vicksburg.
[6] Hallquist, J.O. (2005). Ls-Dyna Theory Manual. Livermore Software Technology Corporation, Livermore.

[7] Dharmasena, K.P., Wadley, H.N.G., Xue, Z., Hutchinson, J.W. (2008). Mechanical response of metallic honeycomb sandwich panel structures to high-intensity dynamic loading. International Journal of Impact Engineering, vol. 35, no. 9, p.1063-1074, D0l:10.1016/j.jijimpeng.2007.06.008.

[8] Cooper, P.W. (1994). Comments on TNT Equivalence. 20th International Pyrotechnics Seminar.

[9] Locking, P.M. (2011). The Trouble with TNT Equivalence. 26th International Symposium on Ballistics.

[10] UFC 3-340-02 (2008). Structures to Resist the Effects of Accidental Explosions. Unified Facilities Criteria.

[11] Bajić, Z. (2007). Determination of TNT Equivalent for Various Explosives. MSc thesis, University of Belgrade, Faculty of Technology and Metallurgy, Belgrade. (in Serbian)

[12] Formby, S.A., Wharton, R.K. (1996). Blast characteristics and TNT equivalence values for some commercial explosives detonated at ground level. Journal of Hazardous Materials, vol. 50, no. 2-3, p. 183-198, D0l:10.1016/0304-3894(96)01791-8.

[13] Simoens, B., Lefebvre, M., Minami F. (2011). Influence of Different Parameters on the TNT-Equivalent of an Explosion. Central European Journal of Energetic Materials, vol. 8, no. 1, p. 53-67.

[14] Sochet, I., Gardebas, D., Calderara, S., Marchal, Y., Longuet, B. (2011). Blast wave parameters for spherical explosives detonation in free air. Open Journal of Safety Science and Technology, vol. 1, p. 31-42, Dol:10.4236/ojsst.2011.12004.

[15] Ackland, K., Bornstein H., Lamos, D. (2012). An analysis of TNT equivalencies using AUTODYN. Journal of Explosion Engineering, vol. 1, no. 3, p. 80-88.

[16] Pachman, J., Matyáš, R., Künzel, M. (2014). Study of TATP: blast characteristics and TNT equivalency of small charges. Shock Waves, vol. 24, no. 4, p. 439-445, D0l:10.1007/ s00193-014-0497-4.

[17] Bogosian, D., Yokota, M., Rigby, S.E. (2016). TNT equivalence of c-4 and pe4: a review of traditional sources and recent data. Proceedings of the 24th Military Aspects of Blast and Shock, Halifax, p. 19-23.

[18] Rigby, S.E., Sielicki, P.W. (2014). An Investigation of TNT Equivalence of Hemispherical PE4 Charges. Engineering Transactions, vol. 62, no. 4, p. 423-435

[19] Locking, P.M. (2011). The trouble with TNT equivalence. 26th International Ballistics Symposium, p. 143-154.

[20] Panowicz R., Nowak J., Konarzewski M., Niezgoda T. (2015). Parametric studies of directed fragmentation warhead used for combat shaped charges. Engineering Transactions, vol. 63, no. 2, p. 181-190.

[21] Chung Kim Yuen, S., Langdon, G.S., Nurick G.N., Pickering, E.G., Balden, V.H. (2012). Response of V-shape plates to localised blast load: Experiments and numerical simulation. International Journal of Impact Engineering, vol. 46, p. 97109, DOI:10.1016/j.ijimpeng.2012.02.007.

[22] Włodarczyk, E. (1994). Introduction to Shock Waves. PWN, Warsaw. (in Polish) 